\author{
MITSUBISHI ELECTRIC RESEARCH LABORATORIES \\ http://www.merl.com
}

\title{
Towards Passive 6D Reflectance Field Displays
}

\author{
Martin Fuchs, Ramesh Raskar, Hans-Peter Seidel, Hendrik Lensch
}

TR2008-098 September 2008

\begin{abstract}
Traditional flat screen displays present 2D images. 3D and 4D displays have been proposed making use of lenslet arrays to shape a fixed outgoing light field for horizontal or bidirectional parallax. In this article, we present different designs of multi-dimensional displays which passively react to the light of the environment behind. The prototypes physically implement a reflectance field and generate different light fields depending on the incident illumination, for example light falling through a window. We discretize the incident light field using an optical system, and modulate it with a 2D pattern, creating a flat display which is view and illumination dependent. It is free from electronic components. For distant light and a fixed observer position, we demonstrate a passive optical configuration which directly renders a 4D reflectance field in the real world illumination behind it. We further propose an optical setup that allows for projecting out different angular distributions depending on the incident light direction. Combining multiple of these devices we build a display that renders a 6D experience, where the incident 2D illumination influences the outgoing light field, both in the spatial and in the angular domain. Possible applications of this technology are time-dependent displays driven by sunlight, object virtualization and programmable light benders / ray blockers without moving parts.
\end{abstract}

ACM Transactions on Graphics 2008

\footnotetext{
This work may not be copied or reproduced in whole or in part for any commercial purpose. Permission to copy in whole or in part without payment of fee is granted for nonprofit educational and research purposes provided that all such whole or partial copies include the following: a notice that such copying is by permission of Mitsubishi Electric Research Laboratories, Inc.; an acknowledgment of the authors and individual contributions to the work; and all applicable portions of the copyright notice. Copying, reproduction, or republishing for any other purpose shall require a license with payment of fee to Mitsubishi Electric Research Laboratories, Inc. All rights reserved.
} 



\title{
Towards Passive 6D Reflectance Field Displays
}

\author{
Martin Fuchs* \\ Ramesh Raskar ${ }^{\dagger}$ \\ MPI Informatik \\ Mitsubishi Electric Research Laboratories \\ Hans-Peter Seidel ${ }^{*}$ \\ MPI Informatik \\ Hendrik P. A. Lensch* \\ MPI Informatik
}

\begin{abstract}
Traditional flat screen displays present 2D images. 3D and 4D displays have been proposed making use of lenslet arrays to shape a fixed outgoing light field for horizontal or bidirectional parallax. In this article, we present different designs of multi-dimensional displays which passively react to the light of the environment behind. The prototypes physically implement a reflectance field and generate different light fields depending on the incident illumination, for example light falling through a window. We discretize the incident light field using an optical system, and modulate it with a 2D pattern, creating a flat display which is view and illuminationdependent. It is free from electronic components. For distant light and a fixed observer position, we demonstrate a passive optical configuration which directly renders a 4D reflectance field in the realworld illumination behind it. We further propose an optical setup that allows for projecting out different angular distributions depending on the incident light direction. Combining multiple of these devices we build a display that renders a $6 \mathrm{D}$ experience, where the incident 2D illumination influences the outgoing light field, both in the spatial and in the angular domain. Possible applications of this technology are time-dependent displays driven by sunlight, object virtualization and programmable light benders / ray blockers without moving parts.
\end{abstract}

CR Categories: I.4.0 [Image Processing and Computer Vision]: General-Image displays I.4.1 [Image Processing and Computer Vision]: Digitization and Image Capture-Reflectance I.3.7 [Computer Graphics]: Three-Dimensional Graphics and RealismColor, shading, shadowing, and texture

Keywords: passive reflectance field display, image-based relighting with natural light

\section{Introduction}

For centuries, there has been technology available to create stained glass - glass which emits a picture by modulating the incident illumination. In this article, we extend this concept to a new kind of displays that renders different images or even light fields depending on ambient illumination. The display for example can show a relightable scene model, rendering this scene in the illumination which is incident to the display from behind (see Figure 1), e.g. in natural lighting outside a window. Illumination dependent effects such as shadows and highlights will change accordingly.

*e-mail:\{mfuchs,hpseidel,lensch $\} @$ mpi-inf.mpg.de

${ }^{\dagger}$ now at MIT Media Lab, e-mail:[raskar]@media.mit.edu

ACM Reference Format

Fuchs, M., Raskar, R., Seidel, H., Lensch, H. 2008. Towards Passive 6D Reflectance Field Displays. ACM Trans. Graph. 27, 3, Article 58 (August 2008), 8 pages. DOI = 10.1145/1360612.1360657 http://doi.acm. org/10.1145/1360612.1360657.

Copyright Notice

Permission to make digital or hard copies of part or all of this work for personal or classroom use is granted without fee provided that copies are not made or distributed for profit or direct commercial advantage and that copies show this notice on the first page or initial screen of a display along with the full citation. Copyrights for components of this work owned by others than ACM must be honored. Abstracting with credit is permitted. To copy otherwise, to republish, to post on servers, to redistribute to lists, or to use any component of this work in other works requires prior specific permission and/or a fee. Permissions may be requested from Publications Dept., ACM, Inc., 2 Penn Plaza, Suite 701, New York, NY 10121-0701, fax + (212) 869-0481, or permissions@acm.org.

$10.1145 / 1360612.1360657$

http://doi.acm.org/10.1145/1360612.1360657
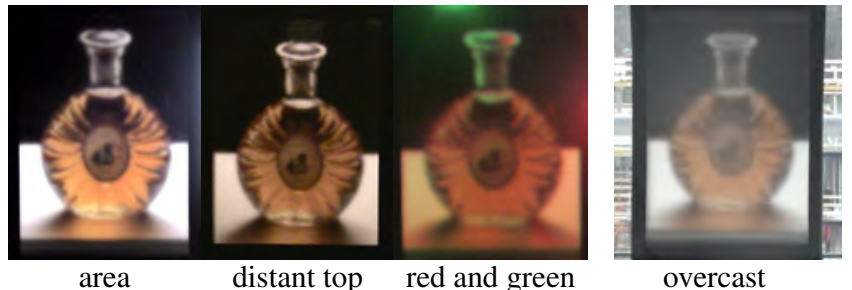

area distant top red and green

overcast

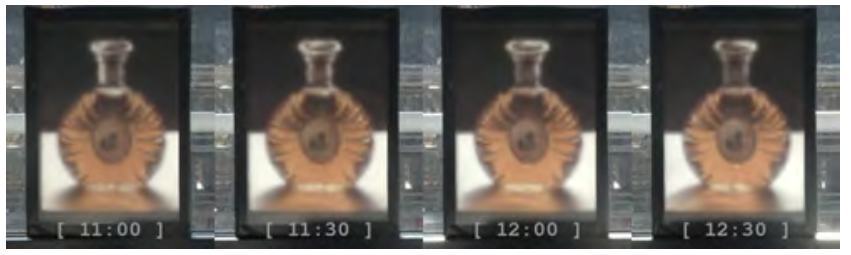

time lapse

Figure 1: A 4D reflectance field display (Design I) illuminated from behind with different incident directions and light source types. For the "time lapse" and "overcast" results, we taped the display to a window and recorded pictures with direct sun illumination and in overcast sky. Note how the highlights on the bottle cap and the shadows move as the illumination direction changes, and how the shadow boundary smoothes for the area light source.

We propose a simple setup which produces these effects by optical means only. Our designs have been inspired by autostereoscopic displays, that produce view dependent images by placing a set of lenses on top of a pattern encoding the outgoing light field. In contrast to autostereoscopic displays, the output of our display depends on the incident illumination (see Figure 2). We describe an optical setup which changes the appearance depending on the illumination angle, and define a mechanism that superimposes the contributions from different angles, effectively computing an expensive integration with optical means.

Using a simple configuration of optical components - mainly lenses and lenslet arrays - we discretize the 4D space of incident light rays, and embed it into a $2 \mathrm{D}$ plane in which a printed pattern performs a direction-dependent modulation. Another set of lenses then shapes the outgoing light field both in the angular or the spatial domain. The pattern can be quickly modified, for the efficient re-use of optical components. Our lens-based designs are considerably more light efficient than designs using slit masks.

In particular, the following designs and prototypes are proposed:

- Design I: a 4D display, producing a 2D image depending on the 2D incident angular light distribution for a fixed observer (Section 4.1),

- Design II: an improvement of Design I for higher precision (Section 4.3)

- Design III: a 4D display that at one location modulates the 2D incident distribution, projecting out a different 2D angular distribution for each incident angle (Section 5.1), and

- Design IV: a 6D prototype which combines multiple 4D displays to modulate the incident light into a 4D light field, varying both in the angular and the spatial domain (Section 5.3).

Both Design I and Design II have deficiencies with respect to con- 


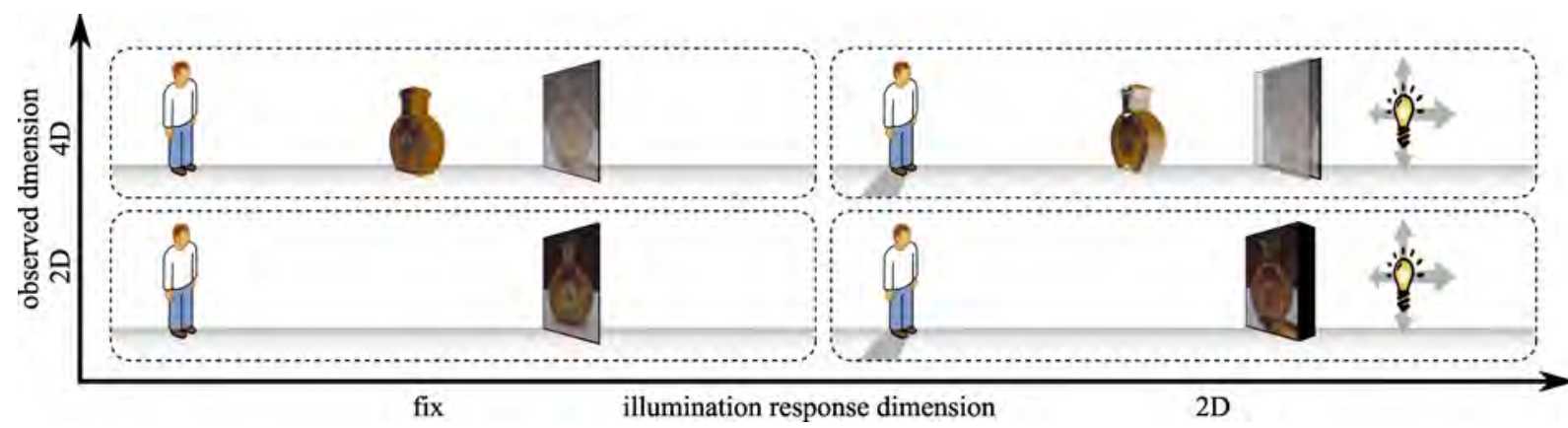

Figure 2: Display technologies: 2D displays are independent from viewer and light. A 4D light field display creates a parallax illusion of an object floating in space, while a $4 D$ reflectance field display changes its flat appearance as its illumination changes. Combined, they become a $6 D$ display: a display which creates a illumination-dependent light field rendering.

trast and transmissivity, which we will discuss in Section 4.2. Still, they demonstrate the concept and are fully applicable in sunlight.

Some of the possible applications of this technology are timevariant transmission in window sheets forming fully passive mood lights using the sun as light source. The displays can show timevariant pictures for natural illuminations. They can act as physical representations of captured 4D reflectance fields and environment mattes, which passively render the object of interest in the ambient illumination using passive optical components only.

\section{Related Work}

\section{Flattening of High Dimensional Visual Data}

High dimensional data structures play an important role in our everyday life: in images, volumes, light fields or reflectance field data sets. However, many optical visualizations and recording techniques are limited to a 2D structure. Therefore, methods have been presented in the past which address this problem by flattening the high dimensional data, embedding it in a planar, 2D representation.

Integral photography [Lippmann 1908] is an early approach which records a 4D light field on a photographic plate. The main concept is adding an array of lenses to the plate, both discretizing the spatial coordinates of a $(\theta, \mathbf{x})$ representation and embedding the angular domain within. In recent years, this approach has been successfully applied to digital recording [ $\mathrm{Ng}$ et al. 2005], with improved lens configurations [Georgiev et al. 2006], for microscopy [Levoy et al. 2006], and with flexible re-parameterization [Isaksen et al. 2000]. Veeraraghavan et al. [2007] have shown that a band-limited light field can also be embedded into sub-bands of the Fourier spectrum.

Light fields can also be encoded in holograms [Ziegler et al. 2007], encoding the multidimensional information in a plane by exploiting phase variations in light wave fronts which are usually not perceived by human observers.

\section{Light Field Displays}

Planar encodings of light fields are since the days of integral photography closely coupled to the development of displays which create a 3D impression by projecting a light field into space. Nakajima et al. [2001] described a lens array on top of a computer display for a 3D viewing experience. In 2004, Matusik and Pfister [2004] presented an end-to-end system which records a 3D light field, streams it over the network and then displays it on a lenticular array screen. Their article also gives a good overview of current multi-dimensional display techniques. Javidi and Okano [2001] discusse a range of related techniques.

\section{Illumination-Variant Displays}

While light fields capture the appearance of a static object, reflectance fields [Debevec et al. 2000] further encode the optical response of an object to illumination. Nayar et al. [2004] presented a display which measures the distant room illumination, approximated as environment map, and interactively renders an image in this illumination. Koike and Naemura [2007] propose an extension towards emitting a light field in a similar fashion. Both displays are electronic and rely on software and hardware evaluating the illumination and rendering the reflectance field. Scharstein et al. [1996] obtained a patent on a device which is passive: it employs optics in order to create a numeral display of the current time. This is achieved by encoding a pattern in a slit mask so that natural sunlight direction produces different symbols. However, this construction inherently blocks the majority of incident light rays.

In this article, we also follow a passive approach to illuminationvariant displays, but, in contrast to Scharstein et al., we use lenses and colored patterns, thus using a larger portion of the available light for a higher contrast display of more expressive patterns.

\section{Overview}

The display types we propose in this article modulate the incident light field both spatially and angularly. This process of modulating an incident light field into a different output light field can be thought of as a particular reflection function, expressed by a reflectance field [Debevec et al. 2000]. In our case, the incident light is assumed to be distant, simplifying the reflectance field to a $6 \mathrm{D}$ function $R\left(\mathbf{x}_{\text {out }}, \omega_{\text {out }}, \omega_{\text {in }}\right)$, which depends on the incident angle $\omega_{\text {in }}$ and the outgoing ray $\left(\mathbf{x}_{\text {out }}, \omega_{\text {out }}\right)$. The mapping from an incident, distant light field $L\left(\omega_{\text {in }}\right)$ to a radiant light field $L\left(\mathbf{x}_{\text {out }}, \omega_{\text {out }}\right)$ is given by

$$
L\left(\mathbf{x}_{\text {out }}, \omega_{\text {out }}\right)=\int_{\Omega} R\left(\mathbf{x}_{\text {out }}, \omega_{\text {out }}, \omega_{\text {in }}\right) \cdot L\left(\omega_{\text {in }}\right) d \omega_{\text {in }} .
$$

Our displays implement the reflectance function physically, using only passive optical elements. They operate on real-world illumination incident from behind the display and transmit a controlled, modified light field.

We present a low-resolution 6D reflectance field display in Section 5.3, and reduce the dimensionality of the reflectance field for the other prototypes by creating the same image for all observer positions (Section 4.1), or causing only angular but no spatial variation (Section 5.1).

All of the different designs make use of common building blocks, which are inspired by previous integral photography approaches. 


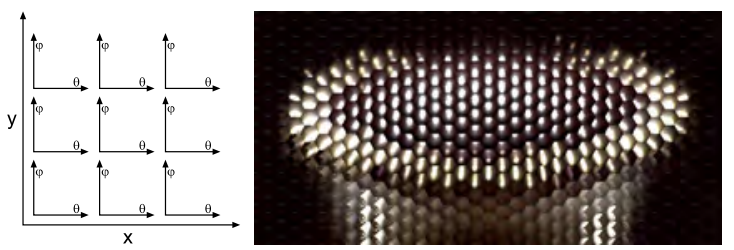

Figure 3: After discretizing, a 4D pattern is embedded into a $2 D$ image (left): macro-pixels capture the variation along the spatial coordinates $(x, y)$, within, micro-pixels vary according to $(\theta, \phi)$. To the right, the cap of the bottle shown in Figure 1 is displayed. $(x, y)$ are distributed according to the hexagonal lattice arrangement of the lenslet array.

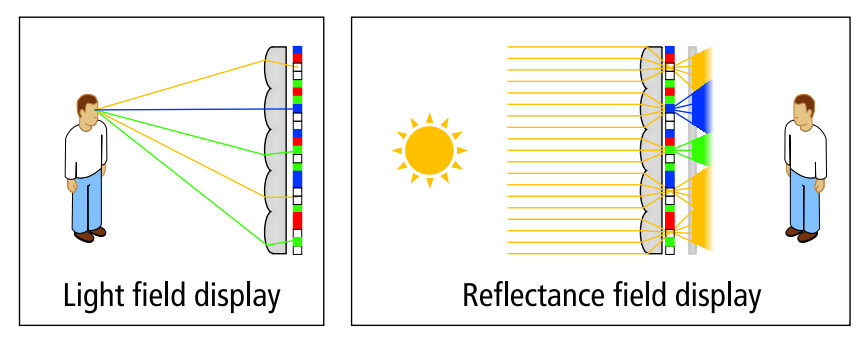

Figure 4: Lens array technology has been used by the integral photography approach to modulate the light field emitted by the display. We propose a modulation according to the illumination angle.

The n-dimensional reflectance function is first discretized and then flattened into a 2D plane, trading the spatial resolution in the plane for encoding both the angular and the spatial variation in various dimensions (see Figure 3).

Each location $p$ within this plane encodes exactly one sample of the reflectance field. Each point is responsible for the modulation of the light transport for one particular incident direction and one outgoing ray $\left(\mathbf{x}_{\text {out }}, \omega_{\text {out }}, \omega_{\text {in }}\right)$. The modulation is carried out physically by letting the incident light field shine through a printed transparency. Printing a different pattern will realize a different reflectance field.

The second common component of our designs is a set of lenses or lenslet arrays which have the task of discretizing and mapping the incident and the reflected light field to the corresponding locations in the plane.

\section{Observer-Invariant displays}

On the way to a full 6D display, we introduce observer-invariant displays (Design I and Design II), that output pictures varying with the incident illumination, but display the same 2D picture for any observer position, providing a 4D viewing experience. Extensions to observer-variant displays will be discussed in Section 5 .

\subsection{Design I}

In order to flatten the 4D reflectance field into a plane, we discretize the spatial dimensions $(x, y)$ into macro-pixels, and, within each macro-pixel, encode the angular variation along $\left(\theta_{i n}, \phi_{i n}\right)$ in micro-pixels, as illustrated in Figure 3.

For guiding the incident light directions to be modulated by the correct micro- and macro-pixel the incident light field is discretized and mapped to this planar representation as well. We apply the same concept as used in integral photography, and use a lens array for this task (see Figure 4). While in traditional light field displays

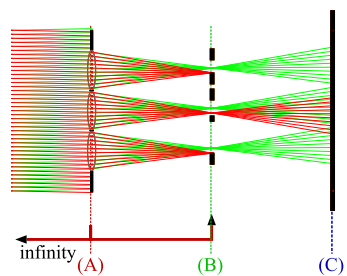

Figure 5: Design I: As the incident light angle changes, the transmissivity of pixels changes. For sufficiently small angles, the individual contributions on the diffuser plane overlap.

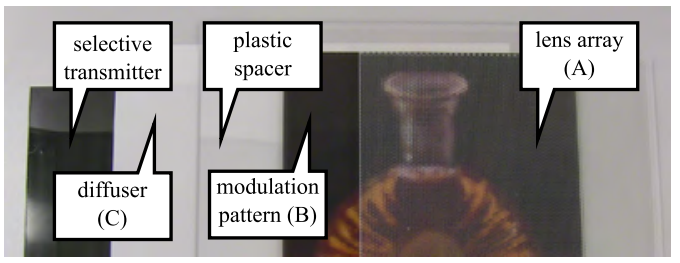

Figure 6: $4 D$ construction for the display from Figure 1, with separated layers. (Design I)

the lenslet array dispatches the different $4 \mathrm{D}$ viewing rays into a $2 \mathrm{D}$ plane we use them to guide the incident light rays to specific locations depending on the light direction. In our case, each lens corresponds to a final macro-pixel, i.e. it will correspond to one pixel as seen by the observer. Assuming a distant, parallel light beam, the lens focuses the light onto a single spot in its focal plane behind the lens. The location of the spot, however, will move depending on the angle of incidence, which is exactly the behavior required to produce the mapping.

The printed transparency is placed in the focus plane. It modulates the color and attenuates the intensity only of that light beam which hits the lens from the corresponding direction. After the modulation the beam again diverges, illuminating a pixel-sized spot on the diffuser surface.

Figure 5 illustrates this design. ${ }^{1}$ A parallel light beam hits an array of lenses (A), which focuses the light on a plane with an embedded pattern (B), flattening the 4D field. Depending on the angular direction and the spatial position, the transparency then modulates the incident light beam with a $R\left(\mathbf{x}, \omega_{i n}\right)$ reflectance field.

In order to provide a view independent experience a diffuser surface (C) is added. It ensures that the modulated beam can be observed from a wide range of viewing angles. Furthermore, it physically integrates over all incoming directions of one macro-pixel as illustrated in Figure 5. Thus, the setup physically evaluates the rendering equation (Eq. 1) in real-time.

\subsection{Results for Design I}

We have implemented a prototype display, which consists of five components (Figure 6): an inexpensive hexagonal lens array (Fres-

\footnotetext{
${ }^{1}$ The ray diagrams are to be read from left-to-right from the light source to the observer. For clarity, we always draw a $2 \mathrm{D}$ cut through the $4 \mathrm{D}$ field and align $x$ with the horizontal axis. In our drawings, we indicate conjugateness for relevant planes by drawing a colored bracket, so that a dotted line indicates the plane where the lens lies, and two arrows indicate the conjugate planes. In cases where one plane is the focal plane of the lens, we label the opposite arrow with "infinity". While in our experiments, we modulate the light field with different colors and varying attenuation, we illustrate the patterns in the ray diagrams either fully transparent or fully opaque in order to prevent additional visual clutter.
} 


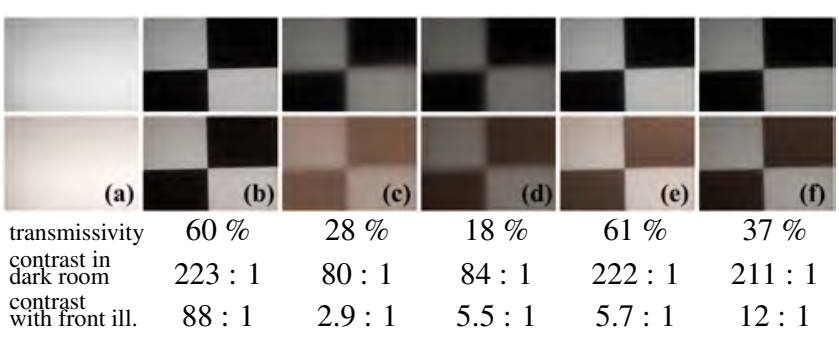

Figure 7: Design I: Transmissivity and contrast experiment: background illumination (a), display with high-contrast pattern without any diffusers $(b)$, with Intellicoat diffuser $(c)$, Intellicoat and $3 M$ (d), holographic diffuser (e), holographic diffuser and $3 M(f)$. Top row: light box in dark room, bottom row: front illumination added.

nel Technologies Inc., 20.77 lenses per $\mathrm{cm}^{2}$, focal length $3 \mathrm{~mm}$ ), a printed transparency (using an Epson Stylus Pro 4800 printer set to $2880 \times 1440$ dpi resolution), a plastic spacer, a diffuser sheet (Intellicoat Technologies DMBF5UV), and a selectively transmissive sheet (see below). In order to improve contrast, we printed a mirrored version on a second transparency and mounted them back-toback, which is a technique occasionally found in commercial printing. Transparent plastic sheets maintain equal distance between the transparency and the diffuser sheet. The distance is chosen to level sharpness and anti-aliasing. We encoded the reflectance field of a bottle into the pattern, which is shown in Figure 3. Details of this process will be found in Section 6 .

Unfortunately, the diffuser screen does not only scatter the illumination along the intended light path, i.e. from the back, but integrates all light from the viewer side as well. This drastically reduces the achievable contrast. This effect can be partially reduced by adding a "Notebook Privacy Computer Filter" by $3 \mathrm{M}$ to the observer side. It is a thin, transparent sheet, which largely reduces transmission for non-normal viewing angles. Still, the best result is achieved with either direct sunlight as back-illumination, or with an artificial light source in an otherwise darkened room. Preliminary experiments with a 30 degrees light shaping holographic diffuser screen from BFi OPTiLAS suggest possible efficiency and contrast gains (see Figure 7). The best material combination will heavily depend on the specific application case.

Figure 1 summarizes the results obtained in various settings, e.g. illuminated with point and area light sources in a darkened lab. The figure further shows the display mounted to an office window for different sky light conditions. As the light direction changes, highlights and shadows of our rendering move in real-time as a result of the optical computation. The point light sources produce crisp and correctly moving highlights. Illuminating with a red and a green light bulb, one sees how the display integrates simultaneously over different light source directions, resulting in separate red or green highlights but rendering the table top in yellow.

The contrast in the window setting is slightly reduced. The overall image resolution is limited to the resolution of the lens array. One can further make out some small blur in the overcast illumination and in the area light source setting. As explained in the next section, without a corrective lens layer, the rendered images tend to shift slightly on the diffuse plane as the incident light direction changes.

\subsection{Design II with Correcting Lenses}

Figure 5 also demonstrates one shortcoming of this simple design. On the diffuser, the modulated beams of one macro-pixel are not perfectly aligned, but only overlap partially. In the worst case, even
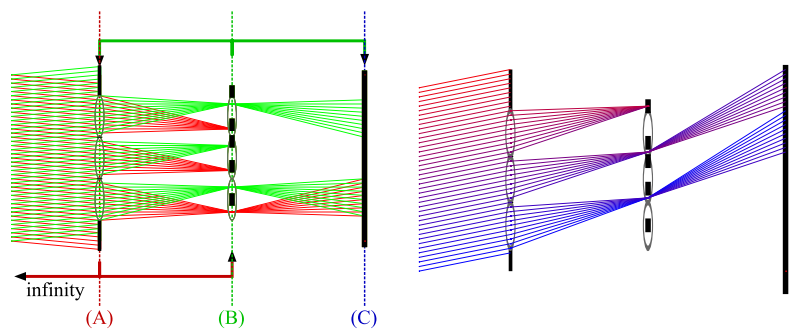

Figure 8: Design II: Adding a lens array with half the focal length of the main lenses in the pattern plane $(B)$ keeps the modulated light cones from moving, generating a precise overlap on the diffuser. For steeper angles, a wrap-over into the next lenslet can still occur.

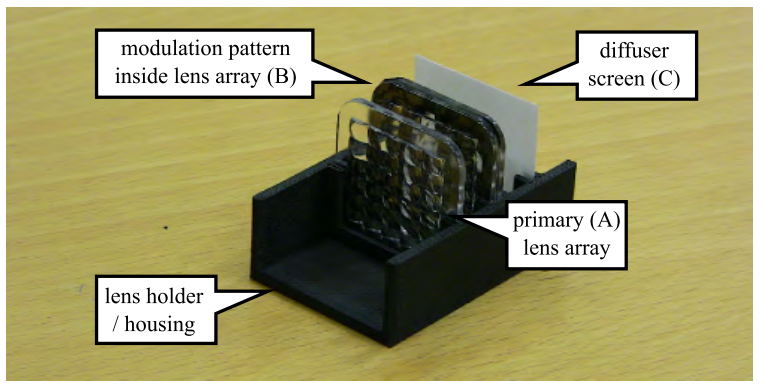

Figure 9: Design II for a small display, with top half of housing removed.

contributions from neighboring macro-pixels are combined. This can be corrected by an improved design (Figure 8), where a second array of lenses is introduced in plane (B). Their task is to project the image of the respective main lens on the diffuser surface. This implies that planes (C) and (A) are conjugate with respect to the corrective lens arrays, and their focal length must therefore be half the focal length of the main lens. If the main lenses are plano-convex, we can re-use parts with the same specification in (B), putting them back-to-back and embedding the pattern in-between.

This additional lens array guarantees a one-to-one mapping between the macro-pixel observed at $(\mathrm{C})$ and its corresponding lenslet in (A). Overlap to a neighboring pixel can only occur if the diffuser plane is moved, or if the incident angle is so steep that the beam through one lens in (A) will hit the neighboring macro-pixel in (B) (see Figure 8).

\subsection{Results for Design II}

The second prototype display includes this corrective lens array. Since it involves more parts of higher optical quality and precision, we have manufactured a prototype of smaller resolution.

The setup is depicted in Figure 9. This time, we use a plano-convex lens array with $25 \mathrm{~mm} \times 25 \mathrm{~mm}$ edge length and $6 \times 5$ lenses of a focal length of $10.5 \mathrm{~mm}$ as primary lenses in plane (A), and two lens arrays of that type as correction layer in plane (B). They are made of glass by Moulded Optics GmbH. We use the same diffuser as in the first prototype. In order to hold the lenses, the pattern and the diffuser precisely in place, a custom-built lens holder has been printed in plastic with a 3D printer. This lens holder also blocks unwanted stray light. As the spatial resolution is much lower than in Design I, we have encoded simpler patterns.

Again, we performed experiments with hand-held light sources in a darkened room. The results are shown in Figure 10. The first row 


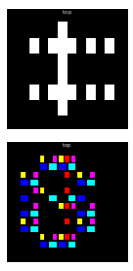

pattern
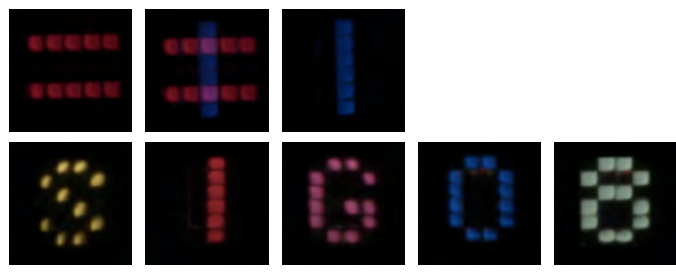

Figure 10: Demonstration of Design II. Top: a simple pattern, demonstrating the exact alignment of the partial pictures. Bottom: a more complicated pattern, illuminated by white light from five different directions.

demonstrates the precise alignment that can be achieved with the corrective lenses: we have created a black and white pattern which shows two horizontal bars when illuminated from the left, and a vertical bar when illuminated from the right. Using red and blue light for the respective directions demonstrates that the individual contributions add up, and the blue and right pixels are precisely aligned. Figure 10 also shows results for a colored pattern which encodes letters for five different illumination directions. When illuminated by white light from different directions, they create the picture of the letters "SIG08".

Some vignetting is noticeable at the borders of the macro-pixels but otherwise the light efficiency is rather good since the light path for every incident direction is controlled by lenses rather than blocking apertures as in the light dependent display of Scharstein [1996].

\section{Observer-Variant Displays}

In the previous section, we have introduced illumination-variant displays which generate planar pictures only. Now, we will show how they can be extended to display different pictures for different viewing angles.

\subsection{Design III}

First, we limit the spatial variation to a single point, describing a design for a display which implements a $4 \mathrm{D}$ reflectance field parameterized by $R\left(\theta_{\text {in }}, \phi_{\text {in }}, \theta_{\text {out }}, \phi_{\text {out }}\right)$. For different incident light directions, it projects out different angular light distributions. Similarly to the previous design, this effect is achieved by mapping each incident light direction into a different spatial location. For each location a different output distribution will be generated by modulation. The proposed design is depicted in Figure 11.

Since the design describes just a single macro-pixel, we put a single big lens in plane (A) where we had the lenslet array in the previous design. The function of this main lens is to map each incident light direction to one particular location on the plane (C). In the example of Figure 11, the incident directions are discretized into three bins which are mapped to three meso-pixels in plane (C).

Instead of modulating the beam in this plane, as we did before, the beam is widened by an array of small lenses in (C). They project the image of the main lens (A) onto the modulation plane (D). For all incident light directions which hit the same meso-pixel this area will be the same, producing the same output distribution. The outgoing distribution is controlled by modulation in plane (D). Each micro-pixel modulates the light for one output direction. As different patterns can be placed beneath each meso-pixel lens, the modulation is different for each incident direction.

The described design does everything necessary to implement a $R\left(\theta_{\text {in }}, \phi_{\text {in }}, \theta_{\text {out }}, \phi_{\text {out }}\right)$ display. As before, we add additional

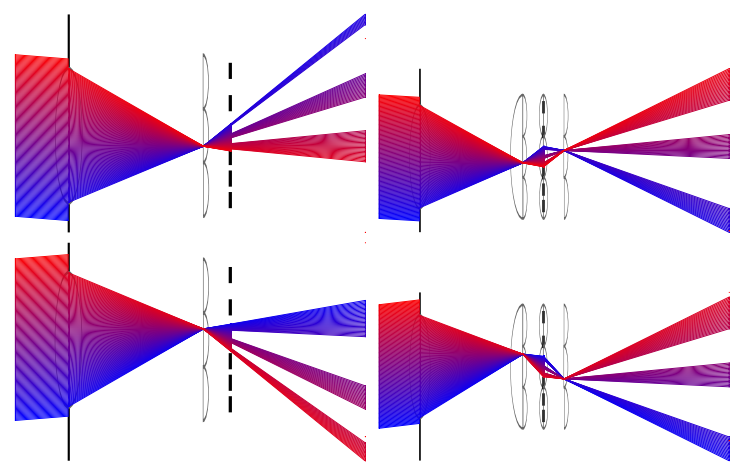

no correction lenses

correction lenses

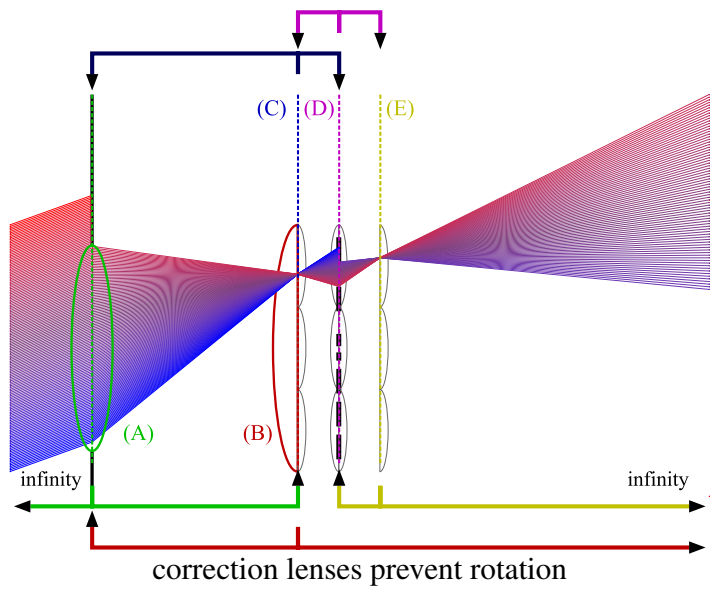

Figure 11: Design III ray diagrams. The simplest design (top left) rotates the outgoing light beams, which is fixed by adding additional lenses (bottom), as depicted on the top right.

lenses to limit the shifting and the rotation of the outgoing distribution which currently slightly changes with the incident angle. We add a field lens (B) in plane (C) with the same focal length as the lens in (A), in order to suppress vignetting. It also rotates the incident light cone at $(\mathrm{C})$ in such a way that its aligns the optical axes of all directions within one meso-pixel. Further, we add a lens array in the pattern plane (D) to project the single spot in the meso-pixel (B) onto a single spot in plane (E). This ensures that each incident light beam of the same bin will undergo the same modulation. Finally, the lens array in (E) widens the beam again for projecting out the angular variation.

This design guarantees that all output distributions of one mesopixel will be perfectly aligned in the angular domain. Spatially they will shift slightly. However, for a large observation distance and a small macro-pixel size, this is hardly noticeable. Under these conditions, the contributions from neighboring meso-pixels, i.e. different incident angles, can hardly be resolved spatially. This effectively accumulates the different output distributions. Given this design of a single pixel which modulates the light in the angular domain, a 6D display can be designed by simply stacking multiple of these macro-pixels in two dimension, thus allowing for spatial and angular light modulation.

\subsection{Results for Design III}

Figure 12 shows a prototype for an observer-variant 4D display, i.e. a single macro-pixel of the $6 \mathrm{D}$ display, which we have again built using a 3D printed lens holder. In its design, we assume that the distance between planes (A) and (B) is large in comparison to 


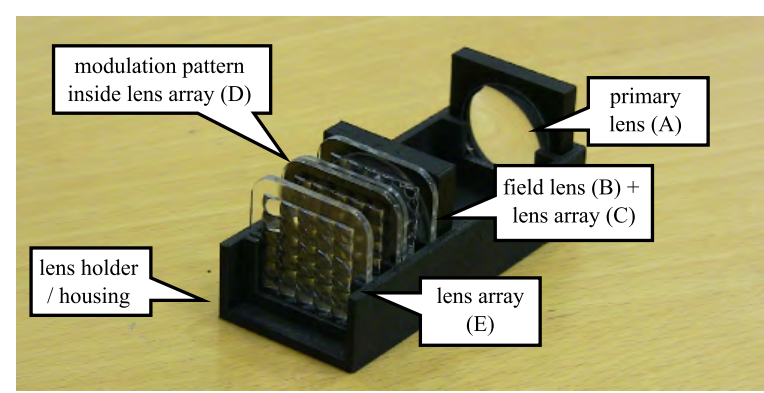

Figure 12: Design III as seen from top with top lid removed.

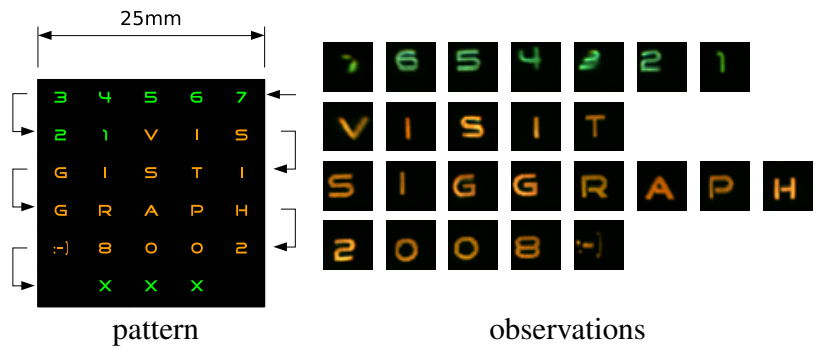

Figure 13: Design III display result: a 4 D projective display, as depicted in Figure 12, projects out different letters in the pattern to the left dependent on the incident light direction. Illuminating the corresponding directions in sequence projects the letters as spatial light distribution, as we have observed on a white receiver surface in the pictures on the right.

the distance between (B) and (D), and choose the distances (B)-(D) and (D)-(E) to be the same. This allows for the use of the same lenslet arrays with focal length $10.5 \mathrm{~mm}$ as used in the prototype of Design 2. In plane (D), the arrays are again arranged in a backto-back configuration to double its focal length. The reuse of the same lenslet arrays in all four places lowers manufacturing costs. Choosing the distance between (A) and (B) to be $50 \mathrm{~mm}$, we can use off-the-shelf coated plano-convex lenses from Edmund Optics Inc. with $50 \mathrm{~mm}$ focal length, and $25 \mathrm{~mm}$ diameter in plane (A) and $30 \mathrm{~mm}$ diameter in plane (B), respectively.

In order to demonstrate the 4D design with angular variation, we put a pattern consisting of digits and letters in plane (D) (see Figure 13), and illuminated the prototype with a distant spot light. The output distribution is made visible by projection onto a diffuser screen which is not directly illuminated by the spot light. We translate the light source, that is, we change the angle of incidence, following the sequence indicated by the small arrows in Figure 13. The result is a time sequence of well readable letters projected onto the screen. In the corners (" 3 " and "7") some vignetting as well as some distortion is noticeable. This could be removed if the field lens would be enlarged to equally cover all meso-pixels in plane (B).

The results in Figure13 share some similarity with the patterns produced by the Design 2 in Figure 10. Design 2 implements an angular-to-spatial reflectance field $R\left(\omega_{\text {in }}, \mathbf{x}_{\text {out }}\right)$ where every output pixel individually depends on the incident light direction. Design III , on the other hand implements an angular-to-angular reflectance field $R\left(\omega_{\text {in }}, \omega_{\text {out }}\right)$. Each letter is projected out by a different meso-pixel. Therefore, the letters are continuous and not composed of sub-pixels.

\subsection{Design IV}

Combining $7 \times 7$ macro-pixels of Design III we built a prototype for a $6 \mathrm{D}$ display implementing $R\left(\omega_{\text {in }}, \mathbf{x}_{\text {out }}, \omega_{\text {out }}\right)$ (Figure 14, De-

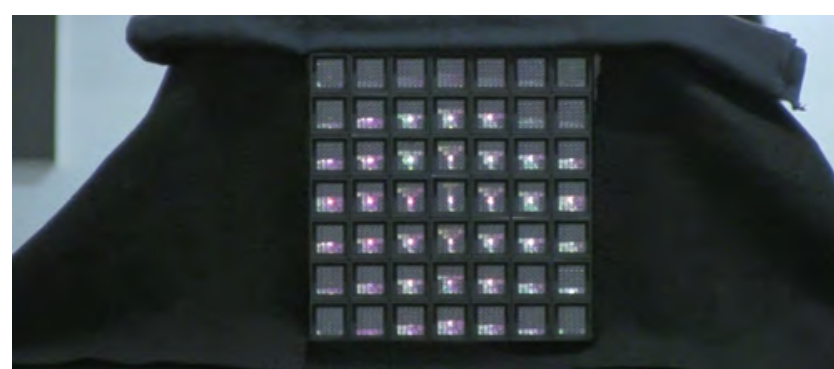

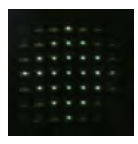

left view
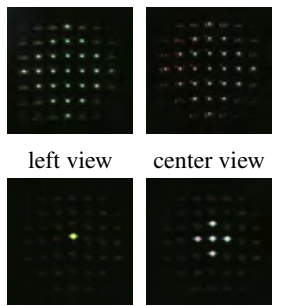

center view

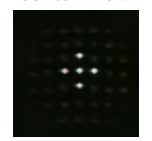

$\longrightarrow$ light source moves from bottom to top $\longrightarrow$

Figure 14: $6 D$ experiment and result (Design IV). Top: fully assembled prototype in mostly diffuse room illumination. The image resembles the pattern from Figure 15. Center row: changing observer direction induces a green to magenta color shift; horizontal light movement empties the patterns inside. Bottom row: vertical light movement induces different patterns. For light movements, we employed focal blur to visualize the effect better.
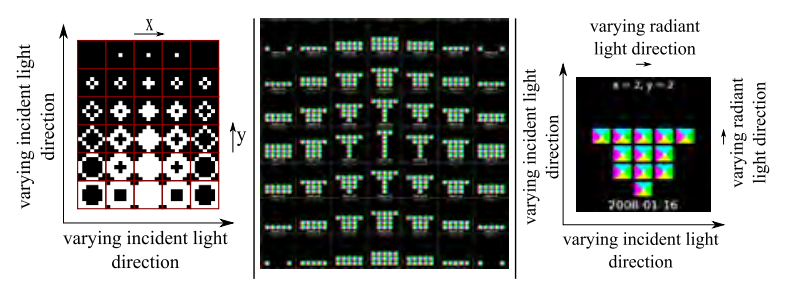

Figure 15: 6D pattern for Design IV. The display shows simple diamond shapes for varying incident directions (left), and adds an angular color modulation, so that they appear magenta when seen from the right, white when seen from the center, and green when seen from the left. This yields the $6 D$ pattern in the center, with a single pixel zoom-in on the right.

sign IV). Every macro-pixel independently produces some output distribution depending on the incident direction. Combined, the display project out different 4D light fields depending on the incident illumination.

\subsection{Results for Design IV}

We created a 6D pattern (see Figure 15) of simple illuminationdependent shapes which grow when the illumination is moved downwards, and appear increasingly hollow when illuminated from the sides. In each output pixel, and for each incident direction we further encode some color variation that depends on the viewing angle. For the sake of simplicity we chose the variation in color to be the same for every macro-pixel. The design does allow for a free configuration though.

In order to produce the results in Figure 14 we surrounded the device by dark cloth and then illuminated and observed the display from distance. In the top image of Figure 14 one actually sees the integration of the patterns produced by the diffuse illumination in the room. At this resolution the structure of the meso-pixels is still 
visible. In the two bottom lines, the device is again illuminated with a hand-held light bulb. The camera is slightly out of focus which makes the light transmitted by the individual meso-pixels more visible. As the camera is moved from left to right the color shift in each pixel can be observed. Moving the light source produces the intended shapes.

Since the observer is not sufficiently distant to the display, we observe not only the white, but also red and green colored light rays, in the pictures for the center view.

\section{Implementation details}

In the previous sections, we have introduced the optical design for our prototypes. Now, we will discuss how to generate the patterns from a reflectance field structure for the 4D case. For our analysis, as well as for the ray diagrams that we have previously shown, we will apply the assumption of paraxial optics, i.e. we assume that the angles of all light rays in our optical system to the optical axes of the lenses within are sufficiently small. In paraxial optics the change of direction and position of each ray due to a lens can be easily modeled with matrix methods [Gerrard and Burch 1975].

The encoding procedure for the pattern is as follows: for each pixel position $\left(p_{x}, p_{y}\right)$ in the pattern

- determine the index $\left(l_{x}, l_{y}\right)$ of the lenslet above the pixel

- determine the pixel position $\left(s_{x}, s_{y}\right)$ relative to the optical axis of the lenslet

- simulate refraction at the lenslet in this pixel for a ray perpendicular to the pattern plane through $\left(s_{x}, s_{y}\right)$, obtaining a ray in direction $(\theta, \phi)$

- color the pixel at $\left(p_{x}, p_{y}\right)$ proportional to $\frac{R\left(l_{x}, l_{y}, \theta, \phi\right)}{\cos \kappa}$, where $\kappa$ is the angle of the refracted ray to the glass surface normal. This factor compensates for less light falling into the lenslet under flat angles.

Finally, we tonemap the pattern by scaling its intensity linearly to a comfortable brightness and performing a gamma and blacklevel correction for the output device. We have tested printers based on laser, ink jet and solid ink technology, and achieved best results with an ink jet printer, putting two sheets back to back for increased contrast.

The pattern used in the demonstrator of Design I in Figure 1 has been computed from a captured reflectance field. It has been recorded with indirect, extended illumination from the entire hemisphere around the object using a setup inspired by [Fuchs et al. 2007]. The display however can only render a smaller range of incident angles. In order to produce a more interesting experience of moving highlights at the top, we have virtually rotated the incident light by 45 degrees during the calculation of the patterns. In principle, one can also re-parameterize the reflectance field along the angular dimension in order to emphasize or suppress the angular dependence.

\subsection{Pattern/Lens Registration}

The pattern and the lenses in our prototype have to be precisely aligned in order to modulate the correct light paths. For Design I, we have first measured the exact lens distance in the lenslet arrays to a higher precision than given by the manufacturer: we have printed patterns for a set of hypotheses and then chosen the distance which minimized the Moiré effects that occur when rotating the lens array on top of the pattern. For precise alignment of the final pattern, we have drilled holes into four lenses at the corners of the lenslet arrays under a microscope, and augmented the pattern by markers which show up below the holes. The patterns for the $6 \times 5$ lenslet arrays used in Design III and Design IV are manually cut and aligned.

\section{Discussion}

\subsection{Limitations}

Our display designs come with some inherent limitations:

For one, we only modulate the light that comes in from the back and project light out to the front side. Light integration is only performed and controlled from one hemisphere. Any light incident from the front hemisphere will limit the contrast to some extend. This is especially true for Designs I and II, which make use of a diffuser which inherently integrates the light from both sides.

Another limitation is given by field of view of the front lenslet array or main lens. The displays currently use much less than the potential 180 degrees of illumination and observation. The same holds for the projection lenses in plane (E) of Design III which produce a rather narrow projection cone.

As we use lenslet arrays without further angular blocking technology, wrapping can occur for rather shallow incident angles. In this case the light beam might cross over to the light path of a neighboring macro-pixel resulting in incorrect and distorted patterns.

Since our display is passive, we can not emit more light from a single macro-pixel than the flux that hits the primary lens. Thus, we cannot display reflectance fields of complicated optical components that focus light from afar on a single pixel with full intensity.

\subsection{Future Challenges}

There is a set of interesting extensions to the presented display prototypes. Most fundamental would likely be an extension to full 8D rendering, with arbitrary incident, non-distant 4D light fields. The design however is not obvious. As long as the light can be assumed distant and 2D parameterizable, we know that each macro lens is hit by the same incident light distribution, and therefore each pattern below the macro lens encodes arbitrarily distant global illumination effects (such as caustics or shadows). For a full 8D display that responds to a 4D incident light field, though, one would need to implement a coupling in the spatial domain. Some means of exchanging energy between different macro-pixels is required in order to faithfully reproduce global illumination effects such as caustics or subsurface scattering. This intrinsically requires a different design.

Another interesting direction of future research might implement the controlled integration of the front hemisphere. The incident light could be reflected back, by a mirror for example. Separate modulation of viewing and illumination rays remains a challenge.

In all the designs we presented, we manually cut out the patterns and aligned them with the optical components. This restricts the precision which we can build the prototypes with and keeps us from shrinking the single pixels, which would be required for higher resolutions. For industrial production, this should be less of an issue.

A principle limit on the resolution of Design III and IV is given by the diffraction limit. Too small features in the modulation plane will widen the transmitted rays resulting in a blurred angular distribution. The diffraction limit is however less a problem for Design I as the resulting image is observed right after the modulation plane.

The presented designs are all based around using lens systems in order to embed high dimensional information in planar surfaces. Holograms exploit variations in the phase of wave fronts; it 
should be interesting to research possible extensions which incorporate holographic techniques for higher data densities and resolutions. Another technology that might influence this area of multidimensional displays is nanotechnology which can already produce surfaces with particularly designed reflection functions [Sun and Kim 2004; Min et al. 2007].

\subsection{Applications}

There is a large set of applications which the presented display designs might enable:

Using Design I we can produce a relightable flattened copy of any $3 \mathrm{D}$ object which will automatically adapt to the incident illumination, providing a relightable object virtualization. This could be useful to present novel products, valuable artefacts, or for instruction manuals in a rather novel way.

Manufacturing Design I at a larger scale one might cover a full window which automatically block or dims the direct sun light according to the time of day, as expressed by the sun position as it follows its arc on the sky. Since the modulation can be both in color and intensity one could also create mood lights.

Inserting a high resolution LCD panel instead of the printed transparencies one can implement even a dynamic reflectance field display. In particular, one could control the transport of individual light rays and dispatch them into different directions.

\subsection{Conclusions}

In this paper, we have presented design and prototypes for a set of different reflectance field displays. They are built using passive devices only, combining lenses and transparencies so as to modulate the outgoing light field depending on the incident light direction. Once built, the displays do not require sophisticated electronics, expensive computations or even electric current.

The proposed designs make use of lenses to guide incident light beams to a particular location in the modulation plane. This design makes use of most of the light that hits the main lens or lens array. Compared to a possible design based on blockers it is inherently more light efficient. While the optical quality of some of the prototypes is currently limited, it can be expected to improve with industrial manufacture. With the presented designs we hope to inspire further exploration and developments of higher dimensional display technology.

\section{Acknowledgements}

We would like to thank our reviewers for all their suggestions and comments, John Barnwell, Douglas Lanman, and Boris Ajdin for help with building the actual prototypes, Conny Liegl and Boris Ajdin for help with the supplemental video, Ankit Mohan for fruitful discussions, and Jay Thornton and Joseph Katz for their support. This work has been partially funded by the DFG Emmy Noether fellowship (Le 1341/1-1), by the Max Planck Center for Visual Computing and Communication (BMBF-FKZ01IMC01), and by Mitsubishi Electric Research Labs.

\section{References}

Debevec, P., Hawkins, T., Tchou, C., Duiker, H.-P., SAROKIN, W., AND SAGAR, M. 2000. Acquiring the reflectance field of a human face. In Proceedings of ACM SIGGRAPH 2000, Computer Graphics Proceedings, Annual Conference Series, 145-156.
Fuchs, M., Blanz, V., Lensch, H. P. A., And SeIdel, H.-P. 2007. Adaptive sampling of reflectance fields. ACM Transactions on Graphics (TOG) 26, 2 (June).

Georgiev, T., Zheng, K. C., Curless, B., Salesin, D., NAYAR, S., AND INTWALA, C. 2006. Spatio-angular resolution trade-offs in integral photography. In Rendering Techniques 2006: Eurographics Symposium on Rendering, 263-272.

GerRARD, A., AND BURCH, J. M. 1975. Introduction to Matrix Methods in Optics. John Wiley \& Sons, New York - Chichester - Brisbane.

Isaksen, A., McMillan, L., And Gortler, S. J. 2000. Dynamically reparameterized light fields. In Proceedings of ACM SIGGRAPH 2000, Computer Graphics Proceedings, Annual Conference Series, 297-306.

JAVIDI, B., AND OKAnO, F. (ED.). 2001. Three-dimensional video and display: devices and systems. SPIE, Washington, USA.

KoIKe, T., AND NAEMURA, T. 2007. BRDF display. SIGGRAPH 2007 poster presentation.

Levoy, M., NG, R., Adams, A., Footer, M., And Horowitz, M. 2006. Light field microscopy. ACM Trans. Graph. (Proc. SIGGRAPH) 25, 3, 924-934.

LIPPMANN, G. 1908. Epreuves reversibles donnant la sensation du relief. Journal of Physics 7, 821-825.

Matusik, W., AND Pfister, H. 2004. 3D TV: a scalable system for real-time acquisition, transmission, and autostereoscopic display of dynamic scenes. ACM Trans. Graph. (Proc. SIGGRAPH) $23,3,814-824$.

Min, C., Wang, P., JiaO, X., Deng, Y., And Ming, H. 2007. Beam manipulating by metallic nano-optic lens containing nonlinear media. Opt. Express 15, 15, 9541-9546.

Nakajima, S., Nakamura, K., Masamune, K., Sakuma, I., AND T., T. D. 2001. Three-dimensional medical imaging display with computer-generated integral photography. Computerized Medical Imaging and Graphics 25, 235-241.

Nayar, S. K., Belhumeur, P. N., And Boult, T. E. 2004. Lighting sensitive display. ACM Trans. Graph. 23, 4, 963-979.

Ng, R., Levoy, M., Brédif, M., Duval, G., Horowitz, M., AND HANRAHAN, P. 2005. Light field photography with a handheld plenoptic camera. Stanford University Computer Scienece Tech Report.

Scharstein, H., Krotz-Vogel, W., and Scharstein, D. 1996. Digital sundial. In US patent 5590 093, German patent 4 431817.

SUN, Z., AND KIM, H. K. 2004. Refractive transmission of light and beam shaping with metallic nano-optic lenses. Appl. Phys. Lett. 85, 652 .

Veeraraghavan, A., Raskar, R., Agrawal, A., Mohan, A., AND Tumblin, J. 2007. Dappled photography: mask enhanced cameras for heterodyned light fields and coded aperture refocusing. ACM Trans. Graph. (Proc. SIGGRAPH) 26, 3, 69.

Ziegler, R., Bucheli, S., Ahrenberg, L., Magnor, M., AND GROss, M. 2007. A bidirectional light field - hologram transform. In Computer Graphics Forum (Proc. Eurographics), D. Cohen Or and P. Slavik, Eds., vol. 26, 435-446. 